\title{
Research and Prospect of Automobile Magneto-rheological Fluid Dual Clutch Transmission
}

\author{
Demin Chen ${ }^{\mathrm{a}}$, Jinhao Xu, Jingwen Pan, Xiaolin Guo, Wei Sun \\ Academy of Armored Force Engineering Institute, Beijing, China \\ admchn@163.com
}

Keywords: magneto-rheological fluid; dual-clutch; MDCT ; research and application

\begin{abstract}
The magneto-rheological fluid Dual Clutch Transmission (MDCT for short) adopts the magneto-rheological (MR for short) effect of magneto-rheological fluid (MRF for short) and the working characters of Dual-Clutch Transmission (DCT for short), and combines the characteristics with long service life, no wear, simple structure, accurate control of MR effect with the principles of DCT technology, it will be a new-style and high-efficiency automatic transmission. This paper elaborates the character, application and the present research of MRF and the traditional mechanistic DCT technology respectively, and put forwards the application prospects of the MRDCT in the future of the automobile automatic transmission.
\end{abstract}

\section{Introduction}

This paper deals with the recent research of the unique and smart material-MRF and the DCT technology. Through the comparison, summary and analysis, finally we put forward the application and development prospect of the MDCT which integrate the unique characteristics of MRF and the structure of DCT technology together. The features and the application potential of such new dual clutch transmission are presented in the following. We believe that it will be a good start.

\section{Feature of the MRF}

In 1948, American scholar Rabinow ${ }^{[1]}$ first invented the magneto-rheological fluid. It mainly contains the non-magnetic fluid and the tiny soft magnetic metal particles with high magnetic conductance, low hysteresis suspended in the fluid evenly. Under the external magnetic field, the MRF could change rapidly and reversibly from the Newton Liquid with a good flow property to a kind of Bingham plastic solid with high viscosity and low liquidity in a milliseconds level of time, and that is called the magneto-rheological effect. Without the external magnetic field, the distribution of the particles is messy, however it will become regular and form a chain-like structure under the magnetic field and the shear stress will increase with the magnetic field. The main performance parameters of MRF are showed in the Table 1.

Table1: The main performance parameters of MRF

\begin{tabular}{|c|c|}
\hline Energy Consumption $\left[\mathrm{J} / \mathrm{cm}^{3}\right]$ & 0.1 \\
\hline Max Field Intensity $[\mathrm{kA} / \mathrm{m}]$ & 250 \\
\hline Max Shear Stress $[\mathrm{kPa}]$ & $50 \sim 100$ \\
\hline Apparent Viscosity $[\mathrm{Pa} \cdot \mathrm{s}]$ & $0.2 \sim 1.0$ \\
\hline Suitable Temperature $\left[{ }^{\circ} \mathrm{C}\right]$ & $-50 \sim+150$ \\
\hline Impurities Sensitivity & Insensitive \\
\hline Density $\left[\mathrm{g} / \mathrm{cm}^{3}\right]$ & $3 \sim 4$ \\
\hline Input Voltage $[\mathrm{V}]$ & $2 \sim 25$ \\
\hline
\end{tabular}

Due to the continuous, reversible, fast reaction, easily controlled, no wear and low consumption characteristics of the MR effect, the MRF devices could be used as the simple, quiet and rapid response intermediate devices in the electrical control system and mechanical system. The MRF and its application devices have attracted extensive attention and interests in the industry and the 
academic circles and it has become an important branch in the current smart materials research ${ }^{[2]}$. In recent several years, the research about MRF and its relative devices has made great strides, not only that we have got the high-quality MRF with stable performance and the yield strength can reach to $100 \mathrm{kPa}^{[3]}$, and the related applications also extended to the valve, sealing, instrumentation, sensors, polishing and automobile, machinery and other engineering field ${ }^{[4]}$. At present, the development of magneto-rheological device research mainly focus on the magnetic fluid active damping shock absorber ${ }^{[5]}$, magneto-rheological valve ${ }^{[6]}$, magneto-rheological polishing ${ }^{[7]}$, engine fan clutch $^{[8]}$, etc. But the research in the automotive clutch which needs to transmit big torque is less and the application technology is still not mature.

MRF devices have three basic work modes: flow mode, shear mode and squeeze mode. The principles of the three basic patterns are shown in figure 1 below $^{[9]}$.

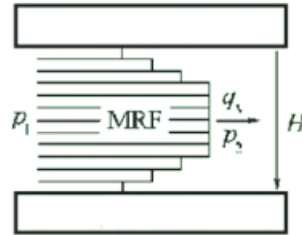

(a)flow mode

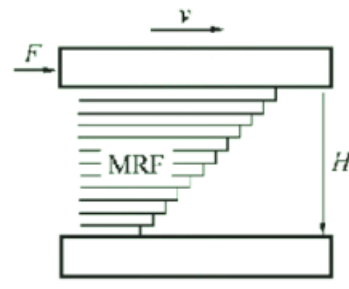

(b) shear mode

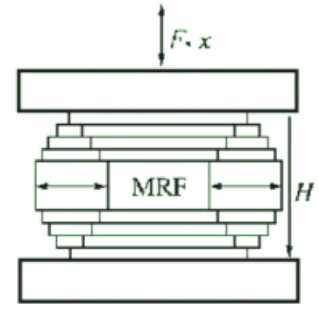

(c) squeeze model

Fig. 1 The three basic work mode of MRF devices

In the flow mode, the MRF is limited to the middle of the stillness magnetic poles, the pressure difference make the flow, the flow resistance is controlled by the magnetic field, the flow mode devices include hydraulic valve, damper (shock absorber) and drivers, etc. In the shear mode, there is relative motion between the poles, this movement makes the MRF being the shear state, the shear stress could be changed by changing the magnetic field intensity, the shear mode devices include brake and clutch, etc. In the squeeze mode, the direction of the magnetic poles' movement is parallel to the magnetic induction line, the status of MRF is alternating stretches and compression, generally speaking, although the displacement of the magnetic poles is small, but that could produce big resistance, it is usually used as vibration damper.

The working principle of the MR clutch is based on the shear mode. Under the external magnetic field, the MRF become the Bingham plastic solid, the magnetic particles are forced to form the magnetic chain which could be connected to the magnetic poles and could prevent the relative movement between the two poles, and that will make the input terminal through the magnetic chain to drive the output terminal; If remove the magnetic field, the magnetic chain will disappear and the two poles is separated again ${ }^{[10]}$. Therefore, we can realize the separation and combination of the MR clutch by the control of the magnetic field intensity.

At present, the research about MR clutch mainly focuses on the aspect of theory, such as the torque transfer model ${ }^{[11]}$, the theory of magnetic circuit design ${ }^{[12]}$, controllable performance of samples, etc. The research and development about production is mainly focuses on the fan clutch ${ }^{[13]}$, etc.

\section{Feature of the DCT}

With the continuous innovation of the automobile technology, the drivers could freed themselves from the frequent and complex shift action, now the vehicles equipped with DCT and also known as the Direct shift Gearbox (DSG for short ) is more and more popular in the customers. The integration of high efficiency, comfort, personality and driving pleasure, the new driving experience, is what the DCT/DSG technology brings us. The structure of Dual-clutch transmission is based on the type of the parallel axis mechanical manual transmission, and it inherited the advantages of the manual transmission such as high transmission efficiency, compact installation space, light weight, low processing cost and etc. What is different, it realized the power shift, not only it ensure the acceleration and security of the vehicle, and as the vehicle will no longer generate the speed instability and impact feeling caused by the shift, thus greatly improve the ride comfort and prolong 
the service life of the drive system ${ }^{[14]}$.

The core component of the DCT is the dual clutch. It is composed of two clutches, the active part is connected to the engine crankshaft or the twist vibration reduction device and the driven parts of the two clutches are connected respectively to the two input shafts (internal and external) of the transmission. The two input shafts are decorated respectively with odd and even gears. In the automobile driving process, through the alternating combination and separation of the two clutches, cooperating the shift control device which is followed to a certain rules, to realize the starting, acceleration and slow down and other working conditions, and to ensure the power shift and the quality of the shift effectively. The structure and transmission principle of DCT are shown in the figure 2 .

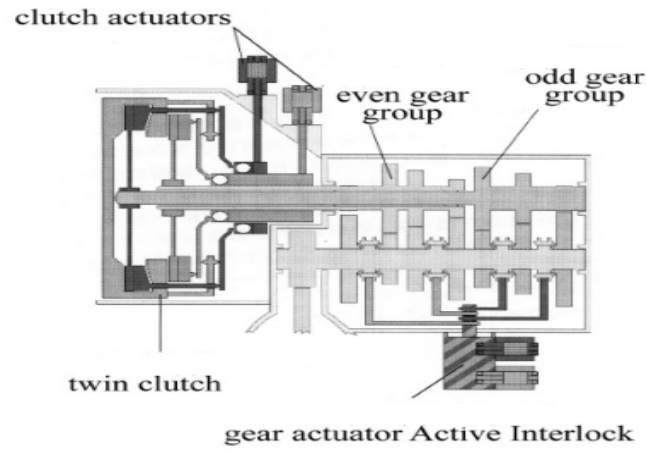

Fig.2 Structure of DCT

Generally, there are two kinds of dual clutches, the wet and the dry. The typical products of the wet dual clutch is produced by the BorgWarner Inc. and it is carried on the 6 speed DSG of the Volkswagen, while the typical products of the dry dual clutch is produced by the Luk in Germany and it is carried on the 7speed DSG of the Volkswagen. The wet dual clutch is filled with oil inside, it could increase the torque transmitted by increase the number of the friction pieces and it always uses the hydraulic or electric devices to control the working pressure of the clutch and to realize the combination and separation, the advantages of the wet dual clutch including the good heat dispersion, the wear of its friction pieces is uniform, work smooth and reliable and more comfort, etc. For the dry dual clutch, it adopts the combination style of two traditional friction clutches, the combination and separation is also operated by the hydraulic or electric devices, the advantages of the dry dual clutch including the high transfer efficiency, the simple structure, the separation is thorough and light weight, etc.

But there is also some certain disadvantages in these two kinds of dual clutches. For the dry dual clutch, the heat dispersion is difficult and the torque that could be transmitted is limited, it is only suitable to be carried on the medium or the small displacement vehicles, in addition, the requirement for the friction pieces is high, the service life is short, the structure is complicated and the requirement for the machining accuracy is high. While for the wet dual clutch, the disadvantage is the higher machining accuracy, the more complicated structure and the higher difficulty and costs of the production.

For the vehicles which are carried with the DCT, no matter it is the wet style or the dry style, the common character is the difficulty of the accurate control to realize the combination and separation in the dual clutch, it`s high requirement for the control system and the relative manipulation system. As a result, the present research about the DCT technology is mainly concentrated on the control and manipulation system. The control system is the main part of the DCT, it has important influence on the starting performance, shift quality, accelerating ability and fuel economy, etc. The core technology of the DCT control system is the starting control strategy, the shift control strategy and shift quality, etc. And the main technology includes: shift time, speed of the clutch combination, tracking quality of the clutch actuating mechanism, overlap quantity and time matching of the alternately work between the two clutches ${ }^{[15]}$ and etc.

\section{Characteristics and Key Technology of the MRF Dual Clutch}




\section{Characteristics of the MRF Dual Clutch.}

(1) No wearing, long service life. Due to the separation and combination of the MRF clutch is realized by changing the viscosity of the MRF. And the characteristics that continuous, reversible and no wear of the MR effect could give the MRF clutch a long service life.

(2) Low energy consumption, Low calorific value. The traditional dual clutch will produce a large amount of heat due to the friction of the friction pieces. If the heat couldn 't be dissipated immediately, the clutch friction surface will produce the local high temperature which will seriously affect the performance of the clutch and the service life. But for the MRF dual clutch, because of the low energy consumption characteristics( only $0.1 / \mathrm{J} / \mathrm{cm}^{3}$ ), there will be little heat during the process of shift, which will be suitable for the long time working of the clutch, and making the clutch has a high reliability and a long service life.

(3) High control accuracy, fast response. Because the reaction process of the MR effect is in the level of milliseconds and the control of the clutch is adjusted by the strength of the magnetic field, while only through adjusting the electric current in the coil, and without the hydraulic control agencies which are used in the traditional wet or dry dual clutch, thus can greatly improve the execution efficiency, reduce the shift time and promote the shift quality.

(4) Simple structure, light weight and low cost. When compared to the wet or dry dual clutch, it is obvious that the structure of MRF dual clutch is simple. Only need to design a reasonable structure, a suitable magnetic circuit layout and good sealing, the function of the clutch will be realized without the hydraulic control system, it is also a good point to improve the market competitiveness.

Key Technology in the Development of the MRF Dual Clutch.

(1) To design a MRF dual clutch which could transmit a certain sizes torque will be the precondition for the realization of the engineering application. According to the present shear yield intensity calculation, it could fulfill the requirement of the torque transmitted for the medium and the small displacement vehicles, on the other hand, the method that increasing the pieces to enlarge the contract area, choosing the quality MRF with high shear yield intensity, enhancing the magnetic field intensity, optimizing the structure of the dual clutch, choosing the reasonable material of the clutch and the working gap between the pieces, etc.

(2) The design of the magnetic circuit. The reasonable design of the magnetic circuit, in some certain circumstance, could acquire sufficient magnetic field intensity, and could transmit sufficient torque.

(3) The reasonable selection and arrangement of the magnetic shielding material. The main function of the magnetic shielding material is to shield the magnetic, to reduce the magnetic flow which form loop without passing the working gap, making sure that the working gap could get enough magnetic field intensity and the sufficient torque could be transmitted. In addition, the two clutches in the dual clutch are nested, in order to avoid the mutual interference between the two clutches, the reasonable arrangement of the magnetic shielding material is necessary for keeping the mutual independence of two clutches.

(4) The reasonable selection of the MRF. Choosing the quality MRF with high performance is a important assurance to realize the every performance index. As the working medium, the MRF need high yield intensity, low viscosity without the magnetic field and good stable performance ${ }^{[16]}$. The ways to solve this problem include that enhancing the suspension performance of the magnetic particles, choosing the suitable additive and the particles with suitable diameter and higher magnetic conductance, etc.

(5) The integral structure design of the MRF dual clutch. Because the automobile clutch has the strict requirement to the size and the weight, not only we should ensure the MRF dual clutch could transmit sufficient torque, it also needs the reasonable size both in axial and radial, and it is a important premise to ensure the MRF dual clutch could be widely applied.

\section{Summary}

Through the research and analysis to the MR character, working principle and the excellent 
control character of the MRF, combined with the structure character and working principle of the automobile DCT and the present barrier in the research and development of the automobile DCT in our country, we put forward the feasibility about the research, development and the vast application prospect of the MRF dual clutch. We believe that along with the increasing requirement about the driving pleasure, the comfort, the energy conservation and the environmental protection of the customers, sooner or later, DCT, this outstanding technology, must will has the shadow of the excellent and perfect smart material-MRF, and certainly the MRF dual clutch also will bring the DCT technology a more broader future.

\section{References}

[1] Rabinow: AIEE Transaction. Forum Vol.67 (1948), p. 1308 1315.

[2] J.X. Wang, G. Meng: Mechanical Strength. Forum Vol.23 (2001), p. 50 56.

[3] P.P. Phule, J.M. Ginder, M. Nakano, Koyama Keds. Proceedings of the 6th International Conference on ER Fluids. Singapore: World Scientific, 1998.

[4] J.P. Ou: Structure Vibration Control (Science Press, Beijing 2003).

[5] S.M. El-Demerdash, D.A. Crolla. Effect of Non-linear Components on the Performance of a Hydro-Pneumatic Slow-active Suspension System. Proc. Instn. Mech. Engrs,1996.

[6] Kordonsky WI: Mater. Sci. Forum Vol. 122 (1993), p. 395.

[7] S.D. Jacobs, F.Q. Yang, E.M. Fess, J.B. Feingold, B.E. Gillman, W.I. Kordonski, H. Edwards, D. Golini. Magneto-rheological Finishing of IR Materials. Proc SPIE, 1997.

[8] Patrick Usoro. Magnetizable Fluid Proves a Plus in a Clutch Situation-new Radiator Fan Drive Boasts the latest in cool[OL]. 2004.

[9] R. Bolter, H. Janocha. In: L.P. Davis, Proc. of SPIE Int. Soc. Opt. Eng. Vol.3045. Washington: SPIE, 1997.

[10] K.J. Jiang, C.Y. Liu: Computer Simulation. Forum Vol.28 (2011), p. 337 341.

[11] J. Zou, J. Huang, Y. Yang: Modern Manufacturing Engineering. Forum Vol.12 (2002), p. 69 70.

[12] G. Yang, B. FSpencer, Jr.J.D. Carlson. Large-scale MR fluid Dampers modeling and dynamic performance considerations. Engineering structures. 2002.

[13] Sevki, Cesmeci, Tahsin Engin: International Journal of Mechanical Sciences. Forum Vol.52 (2010), p. 1036-1046.

[14] C.B. Jing, S.H. Yuan, X.L. Guo: Journal of Mechanical Drive. Forum Vol.3 (2005), p. 56 58.

[15] G.Q.Wu, W.B .Yang, D.T. Qin: Mechanical Engineering. Forum Vol.43 (2007), p. 13 21.

[16] J.X. Wang, G. Meng: Acta Aeronautica ET Astronaut ICA SINICA. Forum Vol.23 (2002), p. $06 \sim 12$. 